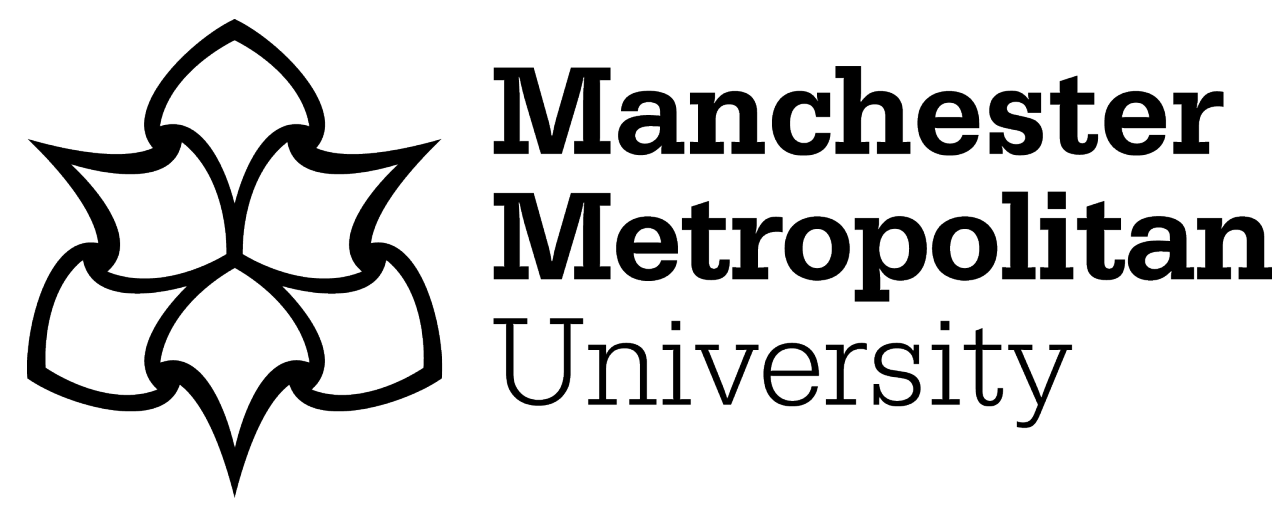

Ahsan, Mominul, Stoyanov, Stoyan and Bailey, Chris (2016) Data driven prognostics for predicting remaining useful life of IGBT. In: 2016 39th International Spring Seminar on Electronics Technology (ISSE), 18 May 2016 - 22 May 2016, Pilsen, Czech Republic.

Downloaded from: https://e-space.mmu.ac.uk/624874/

Publisher: IEEE

DOI: https://doi.org/10.1109/isse.2016.7563204

Please cite the published version 


\title{
Data Driven Prognostics for Predicting Remaining Useful Life of IGBT
}

\author{
Mominul Ahsan, Stoyan Stoyanov and Chris Bailey \\ Computational Mechanics and Reliability Group, University of Greenwich, London, UK \\ Email: M.Ahsan@greenwich.ac.uk
}

\begin{abstract}
Power electronic devices such IGBT (Integrated Gate Bipolar Transistor) are used in wide range of applications such as automotive, aerospace and telecommunications. The ability to predict degradation of power electronic components can minimise the risk of their failure while in operation. Research in this area aims to develop prognostics strategies for predicting degradation behaviour, failure modes and mechanisms, and remaining useful life of these electronic components. In this paper, data driven prognostics approaches based on Neural Network (NN) and Adaptive Neuro Fuzzy Inference System (ANFIS) models are developed and used to predict the degradation of an IGBT device. IGBT life data under thermal overstress load condition with square signal gate voltage bias, available from NASA prognostics data repository, is used to demonstrate the proposed data-driven prognostics strategy. The monitored collector-emitter voltage is used to identify the pattern and duration of different phases in the applied voltage load. The NN and ANFIS models are trained with a subset of the test data to predict remaining useful life (RUL) of the IGBT device under varying load test profiles. The predictive capability and performance of the models is observed and analysed.
\end{abstract}

\section{INTRODUCTION}

The ability to predict failure behaviour of electronic components while in operation can help to take necessary failure preventative actions and to plan for an effective maintenance schedule. Power electronic components such as Insulated Gate Bipolar Transistor (IGBT) play a crucial role in household appliances, automotive, renewable energy, etc. IGBT is increasingly required in many electronic systems with high reliability characteristics, for example in automotive vehicles [1].

The gradual degradation of IGBT decreases the efficiency of an electronic system, and a failure of the device can cause failure of the whole system [1]. Unexpected or sudden failures of IGBT devices occurring in the products or systems can lead to excessive downtime and large losses such as high maintenance cost and lost revenue. There are various failure mechanisms in IGBT that lead to serious failure incident of the products and systems during operation. Bond wire lift off, die solder fatigue, latch up and degradation of substrate are the common extrinsic faults whereas the intrinsic failure mechanisms are dielectric breakdown, hot carrier injection and electro-migration [2, 3]. In this context, it is important to monitor the performance of IGBT during operation, assess the health of the device or module, and plan maintenance activities to avoid catastrophic failure. Diagnosis performs detection, isolation and identification of failure of electronic product where as prognostics is a methodology that can forecast future condition of the electronic product and predict future states and remaining useful life [4].

A number of data driven prognostic approaches such as Particle filters, Kalman filters, Support Vector Machine and other machine learning techniques are being employed for prognostics of electronic components [5]. Xiong et al [6] have proposed a prognostic and warning accelerated test system for IGBTs that are used in electric, hybrid electric and fuel-cell vehicles. In their approach, a computer controlled stress testing is conducted to record parametric measurement and information is used to raise an early failure warning based on detected degradation in the IGBT devices. Temperature and collector-emitter voltage are the used precursor parameters. However, this system is unable to provide the RUL prediction. Algassi et al [7] have proposed a state based prognostics model for predicting remaining useful life of IGBT. K-means clustering, 
evaluations of clustering and RUL calculations are the main computational elements of their approach. In this study, transition probabilities models are used to predict RUL. but overall the system does not provide high accuracy of RUL prediction and high performance when compared to other computational approaches. Sreenuch et al [1] have used Monte-Carlo simulation and collector emitter voltage to develop prognostics algorithm for the RUL prediction of IGBT. Gamma, exponential and Poisson distribution models are combined. The study concluded that the combined model provides better prediction performances compared to the statistical distribution models. However, the system focuses on statistical analysis rather than machine learning.

Currently, significant research efforts are being conducted on prognostics of IGBT with particular emphasis on automotive electronics. However, further work is still required to address many remaining challenges on predicting RUL of IGBT. Hence, an extensive prognostics framework for IGBT is required, underpinned by accurate methods for predicting remaining useful life, to halt expensive sudden failure of these devices.

The aim of this study is to investigate the predictive capability of computational intelligence algorithms such as Neural Network (NN) and Adaptive Neuro Fuzzy Inference System (ANFIS) for predicting RUL of IGBT devices, and more generally of electronic components (IGBT). Results from both approaches are provided in relation to RUL prediction capability. Performances of the two models are compared and reported in the paper.

\section{Proposed RUL Prediction Methodology}

In practice, the IGBT devices have thousand hours lifetime expectancy. However, to analyse degradation, the lifetime of the IGBT is required to be reduced [8]. IGBT accelerated aging system performs robust experiments to investigate prognostics by identifying precursor parameters of the device. In this investigation, the IGBT accelerated aging experimental platform is used for the IGBT aging test developed by NASA AMES laboratory [9]. Then degradation data sets of seven IGBT devices are collected. In the next step, a degradation parameter (collector emitter voltage) for the devices is selected after investigating the data sets. All the degradation data sets are plotted to identify any distinctive degradation pattern. Various degradation phase durations are estimated from the data sets to find out the actual RUL of the IGBT devices. NN is employed to predict RUL of the last device by using the degradation data sets for the first six devices. Subsequently, ANFIS model is also used to find out the prediction of RUL for the same device. Finally a comparison of RUL predictions and errors analysis for both models are conducted. Fig. 1 shows the steps of RUL prediction for IGBT.

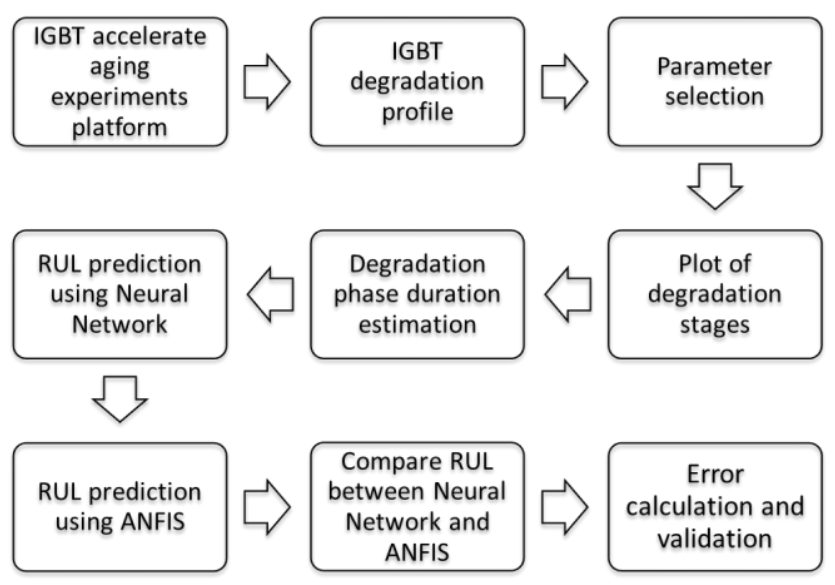

Fig. 1. Process of RUL prediction for IGBT

\section{IGBT DEGRADATION EXPERIMENTS AND DATa Collection}

\subsection{Accelerated aging experimental set-up}

An IGBT accelerated aging experimental system was developed in NASA to explore the degradation process of IGBT for diagnosis and prognosis purposes. The goal of this experiment is to develop a prognostics algorithm for predicting the RUL of IGBT. Fig. 2 shows the experimental set-up for the accelerated aging of IGBT [9].

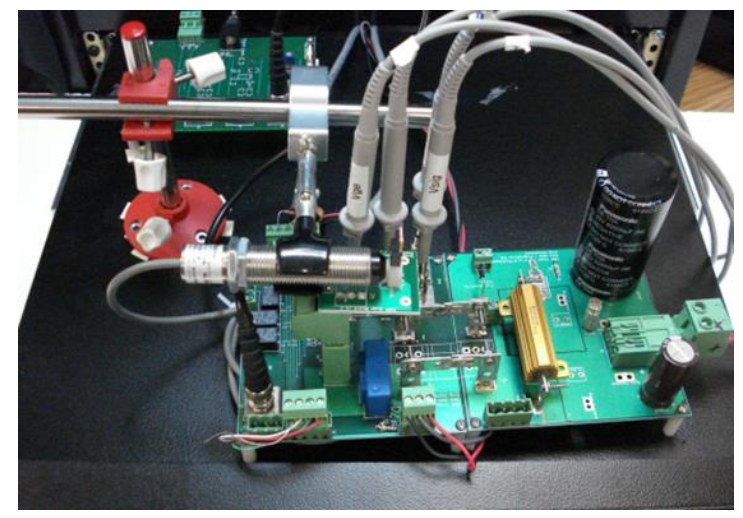

Fig. 2. IGBT accelerated aging test hardware [9]. 
Thermal cycling and electrical overstress are used in this experiment to speed up the degradation and failure of the IGBT. Collector-emitter voltage, collector-emitter current, gate-emitter voltage and gate-emitter current, and environmental parameters such as temperature are considered as the precursor parameters for this aging process [7, 9]. Accelerated aging data sets are collected and recorded from the aging experimental process to assist for further processing of the data in subsequent investigation. The experiment supplies prognostics data sets to develop and compare various data driven algorithms for IGBT prognostics and to estimate RUL.

\subsection{Degradation data}

The IGBT degradation data set is taken from the AMES laboratory of NASA [9]. Tests are carried out on seven IGBT devices using thermal overstress aging condition. Each device is tested with a load profile that results in seven distinctive phases for the measured collector-emitter voltage $\left(\mathrm{V}_{\mathrm{CE}}\right)$. The collector-emitter voltage is considered as a precursor parameter. By controlling the voltage from the power supply, the $\mathrm{V}_{\mathrm{CE}}$ is increased with a step of $0.5 \mathrm{~V}$ from one load phase in the profile to the next phase starting with $2.5 \mathrm{~V}$ at load Phase 1 . The duration of each phase in a full load profile applied to an IGBT varies from device to device and hence affects the failure time. All tested devices failed at some point during the last load phase (Phase 7). An example of a degradation profile of an IGBT device with the measured $\mathrm{V}_{\mathrm{CE}}$ signal is shown in Fig. 3.

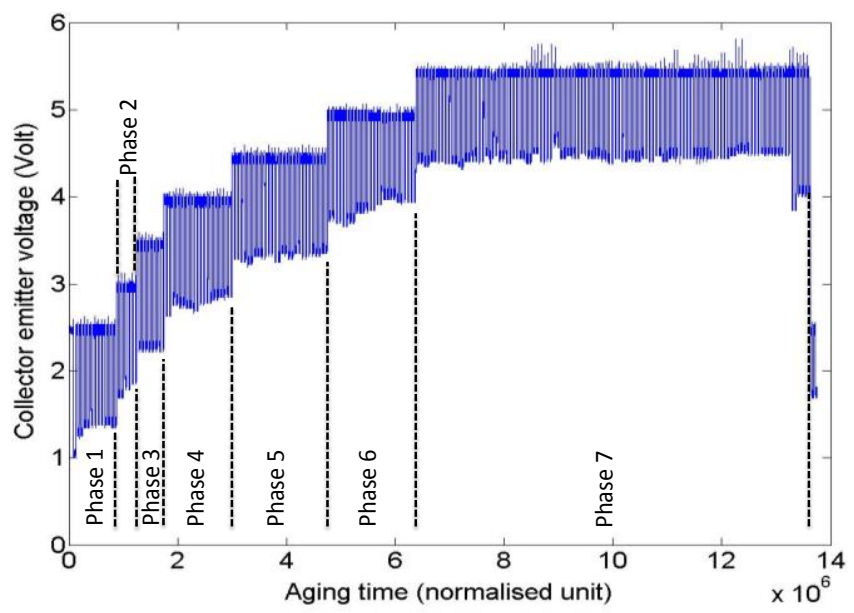

Fig. 3. Degradation profile of collector-emitter voltage for IGBT \#1.

The step-wise profile of the measured $V_{C E}$ indicates clearly the seven phases of the applied test load. Table 1 provides a summary of the durations of the load phases ( 1 to 6 ) for the tested IGBTs. The last column of the table lists the failure time for each of the tested IGBT devices (occurring during the last Phase 7). The run-to-failure degradation process is applied on seven IGBTs under the aging experiment. The data are collected and listed in Table 1 .

Table 1. Duration of load phases and failure time, in normalised time units, identified from $\mathrm{V}_{\mathrm{CE}}$ profiles of the seven tested IGBT devices.

\begin{tabular}{|c|c|c|c|c|c|c|c|}
\hline IGBT & Phase & Phase & Phase & Phase & Phase & Phase & Failure \\
\hline No. & 1 & 2 & 3 & 4 & 5 & 6 & time \\
\hline 1 & 875 & 502 & 645 & 1221 & 1602 & 1454 & 11850 \\
\hline 2 & 1112 & 502 & 1663 & 657 & 1107 & 1725 & 9360 \\
\hline 3 & 1448 & 0 & 1132 & 903 & 712 & 1312 & 10014 \\
\hline 4 & 1225 & 0 & 1160 & 874 & 650 & 1373 & 7864 \\
\hline 5 & 1284 & 424 & 1395 & 683 & 1075 & 3923 & 12068 \\
\hline 6 & 942 & 0 & 1337 & 985 & 1625 & 1012 & 14502 \\
\hline 7 & 1125 & 0 & 872 & 0 & 1237 & 1204 & 6376 \\
\hline
\end{tabular}

\section{Artificial NeURAL NeTWORKS (NN) FOR RUL PREDICTION}

Artificial neural network has high efficiency in prediction purposes. The significance of $\mathrm{NN}$ is arbitrary function approximation and nonlinearity. Furthermore, it also demonstrates effective data relationship and vibrant behavior of systems. The network is consisted of three layers as input, hidden and output layers (see Fig. 4). The performance of NN is considered to predict RUL of IGBT using the degradation data sets.

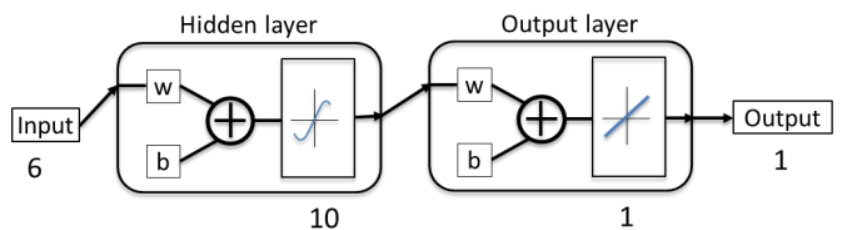

Fig. 4. Neural network (NN) model structure.

\subsection{NN approach: Computational steps}

It is important to prepare different data sets to avoid over fitting of data while training the network. Two different data sets (training and testing) are required to run and validate the model. Six IGBT data 
sets are considered as the training set and the data set for the last device is selected as the testing set. Degradation phase durations are taken as input data of six IGBT devices and the corresponding actual RULs calculated from the phase durations are considered as the target data. Testing of the network is performed after fully completing the training with the network and then RUL is predicted. Fig. 5 shows the computational steps for constructing a NN model using IGBT failure data and predicting RUL of the device.

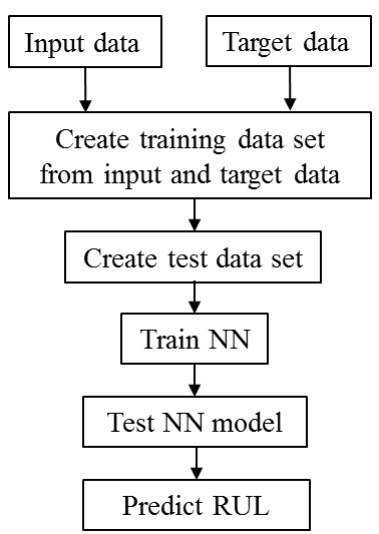

Fig. 5. RUL prediction steps with Neural Network

\section{2. $R U L$ prediction using $N N$ model}

Feed forward $\mathrm{NN}$ is selected for the RUL prediction of IGBT. Levenberg Marquardt (LM) learning algorithm is used to train the network [10]. It is a combination of gradient descent method and the Gauss-Newton method. The algorithm has ability to solve nonlinear problems using its standard technique. Mean squared error evaluates training performance to simplify construction of a network by minimizing sum of the squared errors. The sum of squares is estimated by Hessian matrix as $\mathrm{H}=\mathbf{J}^{\mathrm{T}} \mathbf{J}$ ( $\mathbf{J}$ is Jacobian matrix) and computed gradient as $\mathrm{g}=\mathrm{J}^{\mathrm{T}} \mathrm{e}$ (e is network error). The Levenberg-Marquardt training algorithm is represented by the Eq. 1 .

$$
X_{k+1}=X_{k}-\left[J^{T} J+\mu I\right]^{-1} J^{T} e
$$

where $\mathrm{x}$ represents connection weight, $\mu$ is a scalar combination co-efficient that performs transformation to gradient descent or Gauss Newton algorithm and I stands for Identity matrix [10].

Figure 6 shows the NN model performance in predicting RUL at discrete time points of the test. The predicted values made at earlier times of the test are not accurate and show deviation from the actual
RULs. This is due to the lack of information what the duration of the coming degradation phases will be. Note that the test is predefined with the result in 7 different degradation stages; each causing the collector emitter voltage to increase at certain value but the actual durations of the phases is different in each test. Also, large numbers of data sets are required in order to train the network in recognising likely test duration patterns. Predicted RULs converges to the actual values in the later phases of the test as more and more data on the test history (past degradation phases and their durations) becomes available.

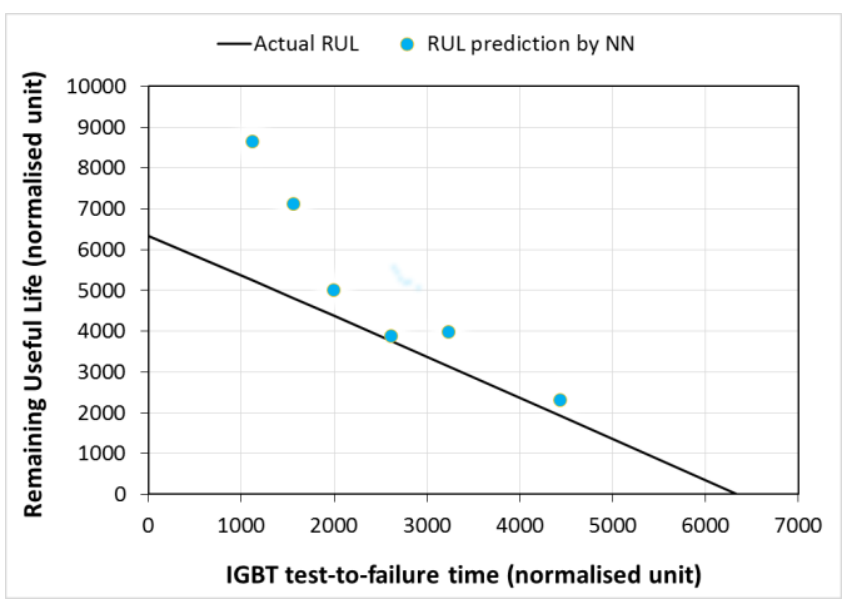

Fig. 6. RUL prediction with Neural Network for an IGBT.

\section{ANFIS FOR RUL PREDICTION}

ANFIS has widely used in fuzzy control, data processing, system identification etc. ANFIS is developed using a fuzzy inference system, fuzzy rules, input and output variables, and membership functions [11]. Takagi-Sugeno rules [12] are used in ANFIS that is developed in this work. The membership functions define the fuzzy sets and calculate degree of membership using the values of inputs. If-then rules are used to obtain output depending on the values of known inputs. A general Sugeno fuzzy model is expressed according to the Eq. 2.

$$
z=a x+b y+c
$$

where $\mathrm{x}$ and $\mathrm{y}$ are two inputs and $\mathrm{z}$ is output [11]. Fig. 7 shows the process of ANFIS RUL prediction for the same device tested by NN. Degradation phase durations and corresponding actual RULs are uploaded as the training data through ANFIS whereas the phase durations of last data sets are uploaded as checking data for the test. ANFIS is configured by six 
inputs and one output with membership functions. Training is progressed after configuring the ANFIS, and then checking is performed and exported the output from the ANFIS model. Finally, ANFIS model is run to predict the RULs from the checking data set.

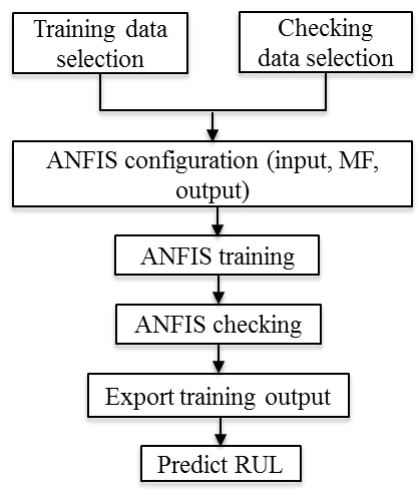

Fig. 7. RUL prediction steps with ANFIS

\subsection{ANFIS model structure}

An ANFIS model is developed. (Fig. 8). The model has six inputs corresponding to the respective phase durations and one output (actual RUL). The model is trained with data points generated using the aging profiles of the first six devices. To improve predictive capability and model accuracy, larger test data sets are required in ANFIS.

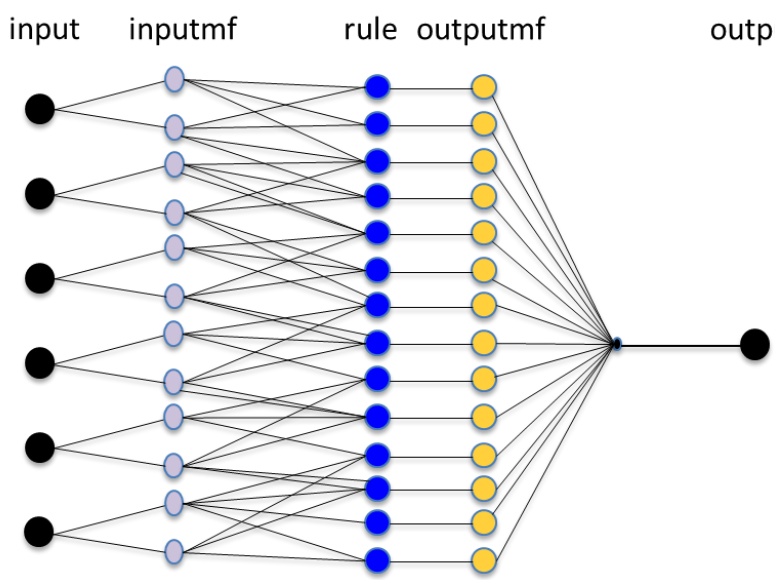

Fig. 8. Representative ANFIS model structure.

\subsection{RUL prediction using ANFIS}

The last tested device, No. 7 in Table 1, is used to check the predictive capability of the models. The RUL predicted by ANFIS are compared with the actual RUL values obtained from the experiment (Fig. 9). Predictions made early in the test are less accurate as the time duration of the coming (future) phases in the full load profile to which the IGBT are yet to be exposed are uncertain. As more information about the past history of the applied load becomes available, the RUL predictions from ANFIS become more accurate.

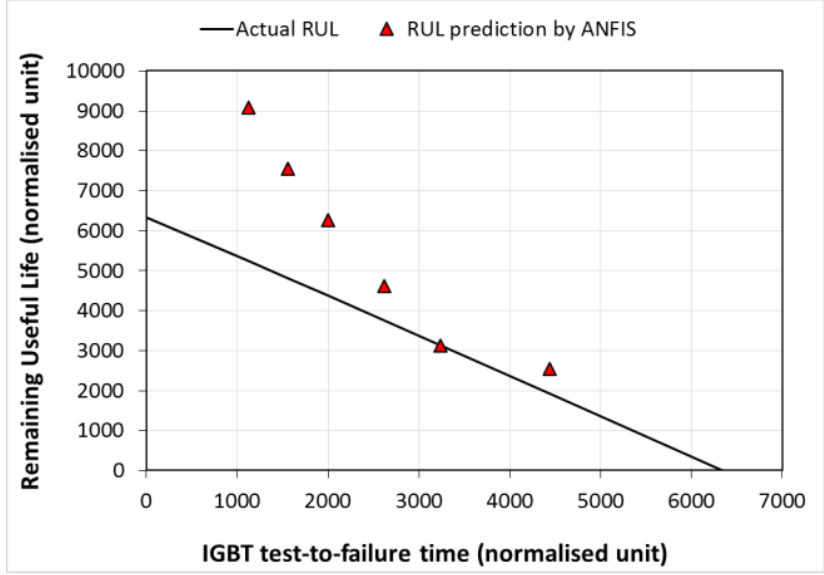

Fig. 9. RUL prediction with ANFIS.

\section{Prediction Comparison ANd VALidation}

Fig. 10 demonstrates the comparison of RUL prediction between NN and ANFIS for the device no. 7. The predictions at the early stages for both $\mathrm{NN}$ and ANFIS showed larger errors. However, the predictions are becoming closer to the actual RULs in the last three stages calculated by both $\mathrm{NN}$ and ANFIS.

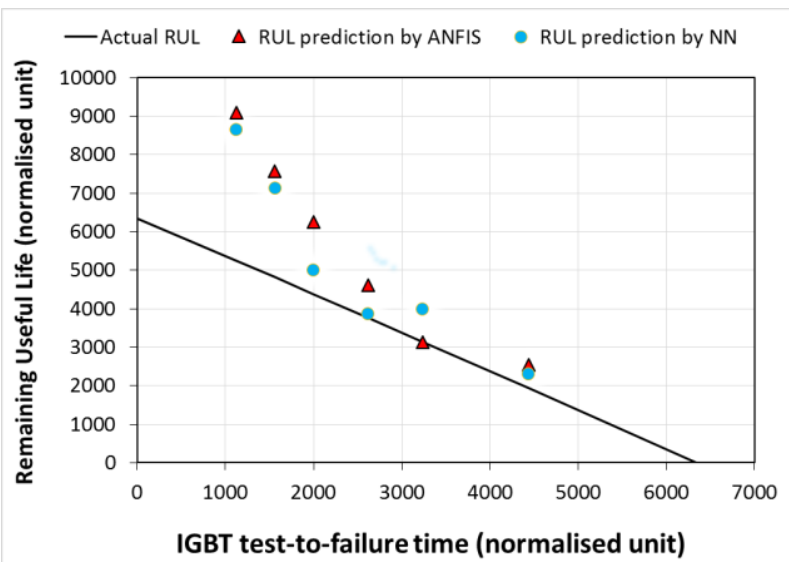

Fig. 10. Comparing RUL prediction between ANFIS and Neural network with actual RUL

In Table 2, RUL prediction errors are drawn for the last phase. The errors calculated using NN and ANFIS are $19.04 \%$ and $30.91 \%$ respectively. In reality, to achieve better prediction, larger data set are required for training purposes in the networks. It is observed that the RULs predicted by NN is slightly more 
accurate compared to ANFIS. Therefore, NN would be better suited to RUL prediction for this type of investigation.

Table 2. Prediction error calculation of RUL using Neural Network and ANFIS for the IGBT No. 7.

\begin{tabular}{|c|c|c|c|c|}
\hline \multicolumn{3}{|c|}{$\begin{array}{c}\text { RUL prediction (normalised } \\
\text { time) }\end{array}$} & \multicolumn{2}{|c|}{ Error } \\
\hline Actual & $\mathrm{NN}$ & ANFIS & NN & ANFIS \\
\hline 1938 & 2307.16 & 2537.20 & $19.04 \%$ & $30.91 \%$ \\
\hline
\end{tabular}

\section{Conclusions}

This study investigated a computational approach for RUL predictions for IGBT modules. The models exploited degradation data obtained from accelerated damage tests with the aim to identify a suitable algorithm that can be useful for predicting accurately the failure time of IGBTs. Data driven approaches that use neural network (NN) and ANFIS models were employed separately and their capability to predict the RUL of an IGBT test device was tested. For both models, it was found that predicted RULs during the early test time phase (initial degradation period) cannot be done accurately due to undefined durations of the future degradation phases in the test. This makes the test condition highly uncertain. However, the accuracy for the predicted RULs gradually improves and started matching the actual RULs as the device goes through and completes more and more of the degradation phases of the test.

A comparison of RUL predictions between NN and ANFIS showed that in the case of the observed test data and test conditions, the proposed NN-based technique resulted in better prediction accuracy than the ANFIS-based technique. On the basis of this investigation, NN-based technique seemed to show better performance and hence judged more appropriate to integrate within prognostics frameworks for evaluating RUL of IGBTs. Further studies on additional test datasets and also using different test profiles are required to gain further insights into the performance of the $\mathrm{NN}$ approach to this problem.

\section{REFERENCES}

[1] Sreenuch, T., Algassi, A. and Perinpanayagam, S. "Probabilistic Monte-Carlo Method for Modelling and Prediction of Electronics Component Life", International Journal of Advance Computer Science and Applications, 2014, vol. 5, no. 1, pp. 96-10

[2] Oh, H., Han, B., McCluskey, P., Han, C. and Youn, B. D. "Physics-of failure, condition monitoring, and prognostics of insulated gate bipolar transistor modules: A review," IEEE Trans. Power Electron., May 2015, vol. 30, no. 5, pp. 2413-2426

[3] Patil, N., Das, D., Goebel, K. and Pecht, M. "Identification of Failure Precursor Parameters for Insulated Gate Bipolar Transistors (IGBTs)," International Conference on Prognostics and Health Management, 2008, Denver, CO.

[4] Tsui, K. L., Chen, N., Zhou, Q., Hai, Y. and Wang, W. "Prognostics and Health Management: A Review on Data Driven Approaches," Mathematical Problems in Engineering, 2015, pp. 1-17

[5] Salunkhe, T., Jamadar, N.I. and Kivade, S.B. "Prediction of Remaining Useful Life of Mechanical Components-A Review", International Journal of Engineering Science and Innovative Technology (IJESIT), 2014, vol. 3, no. 6, pp. 125 - 135

[6] Xiong, Y., Cheng, X., Shen, Z.J., Mi, C., Wu, H. and Garg, V. "Prognostic and Warning System for PowerElectronic Modules in Electric, Hybrid Electric, and Fuel-Cell Vehicles." IEEE Transactions on Industrial Electronics, 2008, vol. 55, pp. 2268-2276.

[7] Alghassi, A., Perinpanayagam, S. and Jennions, I. "A Simple State-Based Prognostic Model for Predicting Remaining Useful," Power Electronics and Applications (EPE), 15 th European Conference, 2013, Lille, France.

[8] Celaya J., Saxena, A., Kulkarni, C., Saha S. and Goebel, K., "Prognostics Approach for Power MOSFET under Thermal-Stress Aging", Reliability and Maintainability Symposium, 2012, Reno, NV

[9] NASA AMES lab, "Prognostics for Electronics," 2015, Available:https://ti.arc.nasa.gov/tech/dash/pcoe/electro nics-prognostics/.

[10]Tian, Z. "An artificial neural network method for remaining useful life prediction of equipment subject to condition monitoring", Journal of Intelligent Manufacturing, 2009, Vol. 23, pp. 227-237.

[11] Alghassi, A., Soulatiantork, P., Samie, M., Perinpanayagam, S. and Faifer, M. "Relibility enhance powertrain using ANFIS base prognostics model," IEEE Conference on Prognostics and Health Management, 2015, Austin, TX, pp. 1-6

[12] Takagi, T. and Sugeno, M. "Fuzzy identification of systems and applications to modeling and control", IEEE Trans. On SMC, 1985, vol. 15, no. 1, pp. 116132. 